\title{
The pion parton distribution function in the valence region
}

\author{
K. Wijesooriya, P.E. Reimer, and R.J. Holt \\ Physics Division, Argonne National Laboratory, Argonne, IL 60439
}

(Dated: September 8, 2005)

\begin{abstract}
The parton distribution function of the pion in the valence region is extracted in a next-to-leading order analysis from Fermilab E-615 pionic Drell-Yan data. The effects of the parameterization of the pion's valence distributions are examined. Modern nucleon parton distributions and nuclear corrections were used and possible effects from higher twist contributions were considered in the analysis. In the next-to-leading order analysis, the high- $x$ dependence of the pion structure function differs from that of the leading order analysis, but not enough to agree with the expectations of pQCD and Dyson-Schwinger calculations.
\end{abstract}

PACS numbers: 14.40.Aq, 25.80.Hp, 12.38.Bx, 12.28.Lg

The pion has a central role in nucleon and nuclear structure. The pion has not only been used to explain the long-range nucleon-nucleon interaction, but also to explain the flavor asymmetry observed in the quark sea in the nucleon. Experimental knowledge of the parton structure of the pion arises primarily from pionic DrellYan scattering [1, 2, 3, 4] from nucleons in heavy nuclei. Recently, the shape of the extracted pion parton distribution function (PDF) at high- $x$, where $x$ is the fraction of the pion momentum carried by the interacting quark, i.e. Bjorken- $x$, has been questioned [5].

The anomalously light pion mass is believed to arise from dynamical chiral symmetry breaking. Any model of the pion must account for its dual role as a quarkantiquark system and the Goldstone boson of dynamical chiral symmetry breaking. Theoretical descriptions of pionic parton structure at high- $x$ disagree. The parton model 6], perturbative quantum chromodynamics (pQCD) 7, 8] and some non-perturbative calculations such as Dyson-Schwinger Equation (DSE) models $[5,[9,10,11,12]$ indicate that the high- $x$ behavior should be near $(1-x)^{a}$, with $a \gtrsim 2$. In contrast, relativistic constituent quark models [13, 14], Nambu-JonaLasinio models with translationaly invariant regularization [15, 16, 17, 18], Drell-Yan-West relation [19, 20] and even duality arguments 21] favor a linear high- $x$ dependence of $(1-x)$. Instanton-based models appear to lie in between these two 22. Lattice calculations yield only the moments of the distributions and not the PDFs themselves [23, 24].

While the PDFs for the nucleon are now well determined by global analyses of a wide range of precise data (see e.g. 25, 26, 27]) the pion PDFs are very poorly known. Presently there are only two experimental techniques to access quark distributions in the pion: the deep inelastic scattering from the virtual pion cloud of the proton for which data is available in the low- $x$, sea region $\left(3 \times 10^{-4} \leq x \leq 0.01\right)[28$, 29], and the DrellYan mechanism which provides data in the valence region $(0.2 \leq x \leq 0.99)$. Unfortunately there is no overlap between these two experimental techniques.

A previous leading order (LO) analysis of pionic DrellYan data by J. Conway et al. (Fermilab E615) [1] sug- gested a high- $x$ dependence of $(1-x)^{1.26}$. The data were at relatively high momentum transfer, $Q^{2}>16(\mathrm{GeV})^{2}$, where pQCD should be a valid approach. Previous analyses in both LO [1, 30] and next-to-leading order (NLO) 31, 32 have adopted a simple functional form for the pionic valence quark distributions or made other assumptions about the parton distributions. Since the original analysis of the Drell-Yan data, it has been suggested 5] that the simple functional form assigned to the valence quarks in the pion and that using only a LO analysis might introduce a bias at high- $x$.

The purpose of this work is to re-analyze the pionic Drell-Yan cross section data in order to study the high$x$ behavior of the pion PDFs and to see if such a bias exists. Cross section data from Fermilab E615 (Conway et al. [1]) were fit to determine the form of the high- $x$ pion parton distribution. A NLO analysis using the well understood PDFs for the proton (MRST98 [26], GRV98 27], and CTEQ5M 25], including nuclear corrections [33]) and allowing for higher twist effects was performed.

The LO Drell-Yan cross section for a pion interacting with a nucleon is

$$
\begin{aligned}
& \frac{d^{2} \sigma}{d x_{\pi} d x_{\mathrm{N}}}=\frac{4 \pi \alpha^{2}}{9 M_{\gamma}^{2}} \\
& \quad \times \sum e^{2}\left[q_{\pi}\left(x_{\pi}\right) \bar{q}_{N}\left(x_{N}\right)+\bar{q}_{\pi}\left(x_{\pi}\right) q_{N}\left(x_{N}\right)\right],
\end{aligned}
$$

where the sum is over quark flavor, $q_{\pi(\mathrm{N})}$ is the PDF for quark flavor $q$ in the pion (nucleon); $e$ is the charge of the quark, $M_{\gamma}$ is the mass of the virtual photon and $x_{\pi(\mathrm{N})}$ is the momentum fraction (Bjorken- $x$ ) of the interacting quark in the pion (nucleon). (Where not needed for clarity, the subscript " $\pi$ " will be dropped.) The cross section per nucleon on an atom with atomic number $Z$ and atomic mass $A$ is

$$
\frac{d^{2} \sigma}{d x_{\pi} d x_{\mathrm{N}}}=\frac{Z}{A} \frac{d^{2} \sigma^{\text {prot. }}}{d x_{\pi} d x_{\mathrm{N}}}+\left(1-\frac{Z}{A}\right) \frac{d^{2} \sigma^{\text {neut. }}}{d x_{\pi} d x_{\mathrm{N}}} .
$$

Charge symmetry (e.g. $u_{\text {prot. }}=d_{\text {neut., }}$ etc.) was then used to express the cross section in terms of just the pion and proton's PDFs. 
The pion's valence $\left(q_{\pi}^{\mathrm{v}}\right)$, sea $\left(q_{\pi}^{\mathrm{s}}\right)$ and gluonic $\left(g_{\pi}\right)$ parton distributions were parameterized as

$$
\begin{gathered}
x q_{\pi}^{\mathrm{v}}(x)=A_{\pi}^{\mathrm{v}}\left[x^{\alpha}(1-x)^{\beta}(1-\epsilon \sqrt{x}+\nu x)\right. \\
\left.\quad+\gamma \frac{2 x^{2}}{9 m_{\gamma \gamma}^{2}}\right], \\
x q_{\pi}^{\mathrm{s}}(x)=A_{\pi}^{\mathrm{s}}(1-x)^{\delta} \text { and } \\
x g_{\pi}(x)=A_{\pi}^{\mathrm{g}}(1-x)^{\eta},
\end{gathered}
$$

respectively. The valence parameterization, $x q_{\pi}^{\mathrm{v}}$, follows that suggested by Hecht et al. 5] with the addition of the term proportional to $x^{2} / m_{\mu \mu}^{2}$ that allows for the possibility of higher-twist effects as suggested by Berger and Brodsky [34] and used by Conway et al. 1, 2]. The coefficient of this term, $\gamma$, was restricted to only positive values. This parameterization reduces to the "minimal parameterization" used in earlier works [1, 2] if $\epsilon$ and $\nu$ are both fixed at 0 . If $\beta=1$, the pion valence PDF has a linear high- $x$ behavior and as $\beta$ increases, there is more curvature at high- $x$. The pion's sea and gluon distributions are identical to those used in earlier works. We assume the pion's valence and sea distributions are $\mathrm{SU}(3)$ flavor symmetric:

$$
\begin{aligned}
x q_{\pi}^{\mathrm{v}}(x) & =x \bar{u}_{\pi}^{\mathrm{v}}(x)=x d_{\pi}^{\mathrm{v}}(x) \text { and } \\
x q_{\pi}^{\mathrm{s}}(x) & =x \bar{u}_{\pi}^{\mathrm{s}}(x)=x u_{\pi}^{\mathrm{s}}(x) \\
& =x \bar{d}_{\pi}^{\mathrm{s}}(x)=x d_{\pi}^{\mathrm{s}}(x) \\
& =x \bar{s}_{\pi}^{\mathrm{s}}(x)=x s_{\pi}^{\mathrm{s}}(x) .
\end{aligned}
$$

The sea and gluonic distributions are better determined from other data. The shape of the gluon distribution is deduced from CERN WA70 prompt photon data 35]. A fit to these data is it best described by $\eta=2.1$ and $G_{\pi} \equiv$ $\int_{0}^{1} x g_{\pi}(x) d x=0.47[31]$. The sea distributions can be determined by comparing $\pi^{+}$and $\pi^{-}$induced Drell-Yan scattering as was done by the CERN NA3 collaboration who found $\delta=8.4$ 3]. These parameters were fixed at the values given above and were also used by Conway et al. 1].

Sum rules constrain the normalization coefficients $A_{\pi}^{\mathrm{v}}$ and $A_{\pi}^{\mathrm{s}}$. Specifically, the total number of valence $\bar{u}$ (and d) quarks in the $\pi^{-}$is constrained to be unity by

$$
\int_{0}^{1} q_{\pi}^{\mathrm{v}}\left(x_{\pi}\right) d x_{\pi}=1
$$

Momentum conservation within the pion is enforced by requiring

$$
2 \int_{0}^{1} x q_{\pi}^{\mathrm{v}}\left(x_{\pi}\right) d x_{\pi}+6 \int_{0}^{1} x q_{\pi}^{\mathrm{s}}\left(x_{\pi}\right) d x_{\pi}+G_{\pi}=1 .
$$

Previous experiments have shown that the basic, LO Drell-Yan cross-section formula fails to explain the magnitude of the observed cross-section, $\sigma^{\exp }$, by a factor of nearly two. This is traditionally accounted for by

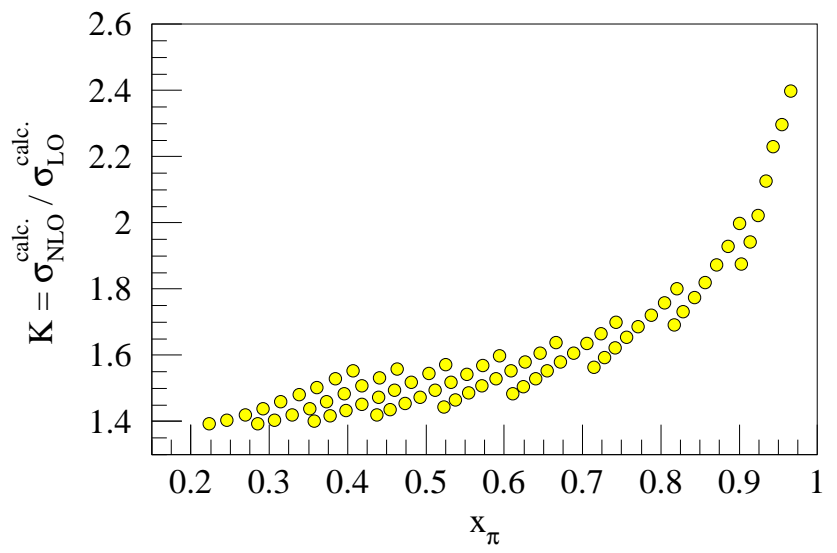

FIG. 1: (Color online) The ratio of NLO to LO pionic DrellYan cross sections ( $K$-factor) calculated using CTEQ5M proton PDF and the pion PDF from this work. One point is shown for each $\left(x_{F}, \sqrt{\tau}\right)$ bin projected onto the $x_{\pi}$ axis. NLO terms are clearly important as $x \rightarrow 1$.

multiplying the LO cross section, $\sigma^{\mathrm{LO}}$ by a " $K$ "-factor, i.e. $\sigma^{\exp }=K \sigma^{\mathrm{LO}}$, which is completely independent of kinematics. This difference is attributed to NLO terms and in proton-nucleon Drell-Yan, $K^{\prime} \approx 1$, where $\sigma^{\exp }=K^{\prime} \sigma^{\mathrm{NLO}} 36$. Comparisons of LO and NLO cross section calculations show that the " $K$ "-factor is not completely independent of kinematics, in particular in the high- $x$ region, as shown in Fig. 1 Note that $K$ and $K^{\prime}$ are indistinguishable from normalization errors. In the NLO fits, it was assumed that the NLO calculation accounted for the entire observed cross section, and so $K^{\prime}$ reflected the normalization uncertainty. Thus, deviation of $K^{\prime}$ from one contributed to the $\chi^{2}$ of the NLO fits, while $K$ was allowed to vary freely in the LO fits.

The differential cross-section measured by Conway et al. 1] were the basis for this work. Events arising from $J / \psi$ and $\Upsilon$ decays were removed by requiring that $4.05 \leq$ $M_{\gamma} \leq 8.53 \mathrm{GeV}$. (Relaxing this condition to include bins with $M_{\gamma} \geq 11.06 \mathrm{GeV}$ had no noticeable effect on the results.) Only bins with $x_{F} \geq 0$ (Feynman- $x$ ) were included in the fit. Furthermore to minimize the effects of shadowing in copper, only bins in which $x_{\mathrm{N}} \geq 0.06$ were used. Finally, if the center of the bin was not within the defined acceptance that bin was not considered by the fit. It is important to note that this final restriction only removed six bins; however, three of these had $x_{\pi} \geq$ 0.97 . These same restrictions were used in the original fit of these data 1, 2]. With these restrictions, data from 76 bins of the 168 tabulated in Ref. 1] remained. The averaged mass for the data included in the fit was 5.2 $\mathrm{GeV}$.

The data were fit in both NLO and LO. In both cases, no QCD evolution was performed so that the resulting pion PDFs are at the average mass scale of the data, $\left\langle M_{\gamma}\right\rangle^{2}=(5.2 \mathrm{GeV})^{2}$. The LO fits were used to compare with the fit of Conway et al. 1] and so that the 
TABLE I: Tabulated below are the results from the leading order (LO) and next-to-leading order (NLO) fits at a mass scale of $\left\langle M_{\gamma}\right\rangle^{2}=(5.2 \mathrm{GeV})^{2}$. The original LO fit of Conway et al. [1] is tabulated in the first column. The second column is a LO fit using the Conway et al. 1] parameterization of the proton. Succeeding three columns are based on the CTEQ5L parameterization of the proton showing the effects of nuclear corrections and the extended pion PDF parameterization. Following the LO results are the results of the NLO fits. The sixth column gives the results for the full parameterization while the seventh column shows the "minimal" parameterization results (preferred fit). The next two columns give the results for the "minimal" parameterization using the MRST and GRV proton parameterizations. In the final column we see the effect of eliminating the higher twist contributions. In all cases, $\delta$ and $g_{\pi}$ were fixed at $\delta=8.40$ and $g_{\pi}=0.47$.

\begin{tabular}{|c|c|c|c|c|c|c|c|c|c|c|}
\hline \multirow{3}{*}{$\begin{array}{l}\text { Prot. PDF } \\
\text { Nucl. Corr. }\end{array}$} & \multicolumn{5}{|c|}{ Leading Order Fit Results } & \multicolumn{5}{|c|}{ Next-to-Leading Order Fit Results } \\
\hline & Fit from & Conway & CTEQ5L & CTEQ5L & CTEQ5L & CTEQ5M & CTEQ5M & MRST98 & GRV98 & CTEQ5M \\
\hline & Conway [1] & - & - & EKS98 & EKS98 & EKS98 & EKS98 & EKS98 & EKS98 & EKS98 \\
\hline$\alpha$ & $0.60 \pm 0.03$ & $0.65 \pm 0.07$ & $0.67 \pm 0.07$ & $0.67 \pm 0.07$ & $0.43 \pm 0.17$ & $0.43 \pm 0.05$ & $0.70 \pm 0.06$ & $0.69 \pm 0.06$ & $0.69 \pm 0.06$ & $0.66 \pm 0.04$ \\
\hline$\beta$ & $1.26 \pm 0.04$ & $1.33 \pm 0.08$ & $1.35 \pm 0.08$ & $1.36 \pm 0.08$ & $1.38 \pm 0.26$ & $1.60 \pm 0.08$ & $1.54 \pm 0.08$ & $1.54 \pm 0.08$ & $1.53 \pm 0.08$ & $1.46 \pm 0.04$ \\
\hline$\epsilon$ & 0.00 & 0.00 & 0.00 & 0.00 & $-3.39 \pm 7.02$ & $-3.30 \pm 1.90$ & 0.00 & 0.00 & 0.00 & 0.00 \\
\hline$\nu$ & 0.00 & 0.00 & 0.00 & 0.00 & $-0.76 \pm 5.52$ & $-0.01 \pm 1.35$ & 0.00 & 0.00 & 0.00 & 0.00 \\
\hline$\gamma$ & $0.83 \pm 0.26$ & $0.78 \pm 0.65$ & $0.75 \pm 0.63$ & $0.77 \pm 0.62$ & $3.0 \pm 2.8^{a}$ & $3.0 \pm 2.0^{a}$ & $0.60 \pm 0.34$ & $0.57 \pm 0.34$ & $0.57 \pm 0.34$ & 0.00 \\
\hline$K\left(K^{\prime}\right)$ & $1.75 \pm 0.13$ & $1.57 \pm 0.11$ & $1.53 \pm 0.10$ & $1.49 \pm 0.10$ & $1.56 \pm 0.24$ & $1.02 \pm 0.05$ & $0.97 \pm 0.06$ & $0.98 \pm 0.06$ & $0.98 \pm 0.06$ & $1.01 \pm 0.05$ \\
\hline$\chi^{2} / N_{D F}$ & $359 / 329$ & $67.6 / 72$ & $67.1 / 72$ & $69.3 / 72$ & $69.1 / 70$ & $72.8 / 70$ & $73.1 / 72$ & $72.0 / 72$ & $70.9 / 72$ & $74.4 / 73$ \\
\hline
\end{tabular}

${ }^{a}$ Converged at upper limit of parameter.

effects of the NLO terms in the cross section could be clearly understood. The results shown in Tab. [1 All of the LO fits have slightly more curvature at high- $x$ than the original fit of these data [1]. This includes the one that used the Conway parameterization of the proton. The original work by Conway et al. was based on a finer binning of the data, but the largest differences between the two fits are in the $K$-factor. In this context, it should be noted that a direct calculation based on Conway et al.'s parameterizations of the proton and pion reveal that $K=\sigma_{N L O} / \sigma_{L O} \approx 1.5$ in close agreement with the present fit and a kinematic dependence similar to that in Fig. 11 Fixing $K=1.75$, more closely reproduces Conway et al.'s fit with $\alpha=0.56 \pm 0.01$ and $\beta=1.24 \pm 0.05$. The use of modern parton distributions and the inclusion of nuclear corrections have only a slight effect on the high- $x$ PDF. In LO when $\epsilon$ and $\nu$ were allowed to freely vary there were significant correlations between fit parameters with $\epsilon$ becoming large and negative while $\gamma$ became large and positive for trivial gains in $\chi^{2}$. A bound of $0<\gamma<3.0$ was implemented in the fit. The shallowness of the $\chi^{2}$ hyper-surface is evident in the large uncertainties in these parameters.

These fits were repeated in NLO using the CTEQ5M proton parameterization. As expected from the kinematic dependence of the $K$-factor (see Fig. (1) there is even more curvature at high- $x$ in both the "minimal" and full parameterization with $\beta=1.54$ and $\beta=1.60$ respectively. As in the LO case, the fit to the full parameterization was able to offset increases in $\gamma$ (the higher twist term) with changes in $\epsilon$ and $\nu$ for extremely slight gains in $\chi^{2}$. Based on this, and the relatively large uncertainties in these three parameters, it was reasonable to remove either $\gamma$ from the fit or fix $\epsilon=0$ and $\nu=0$. Fixing $\gamma$ at values between 0.0 and 0.8 yields fits which have significantly larger uncertainties in $\epsilon$ and $\nu$. Alternatively, $\gamma$ has a direct physical interpretation, while $\epsilon$ and $\nu$ dilute the interpretation of $\beta$ and are merely present to allow for a better representation of the data. While not considered in this work, the angular distributions observed by Conway et al. and by S. Falciano et al. (CERN NA10) 37] are better described with the inclusion of the higher twist term. Using the "minimal" parameterization (fixing $\epsilon=0$ and $\nu=0$ ) and allowing $\gamma$ to vary fits the data as well as allowing all three parameters to vary. In fact, the distributions of residuals as a function of $x$ are nearly identical to the full fit. Based on these considerations, the "minimal" parameterization was adopted as the preferred parameterization. This fit, compared to data is shown in Fig. 2] and the resulting pion valence parton distribution in Fig. 3 To facilitate the comparison of this fit with lattice results, Tab! gives the moments, $\int_{0}^{1} x^{n} q_{\pi}^{\mathrm{v}}(x) d x$, of the pion's valence distributions.

To determine if there was any bias in the fit, it is useful to examine the residual or pull of each data point defined by $R=\left(\sigma^{\text {meas. }}-\sigma^{\text {calc. }}\right) / \delta^{\text {meas. }}$ where $\sigma^{\text {meas. (calc. })}$ is the measured (calculated) cross section and $\delta^{\text {data }}$ is the uncertainty of the measured cross section. The distribution of the residuals as a function of $x$ reveals no bias, shown in Fig. 4 The overall distribution is well described by a Gaussian distribution with a standard deviation consis-

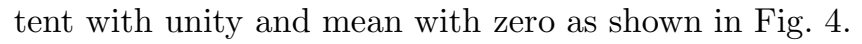

For comparison of the influence of the proton PDF on the fit, additional NLO fits were performed using the MRST [26] and GRV98 27] parameterizations. These fits produced results similar to those obtained using the CTEQ parameterization, as shown in Tab. I

The contribution of the pion's strange sea was also investigated. The strength of the pion's strange sea was varied from being equal to the up and down sea $[\mathrm{SU}(3)$ 

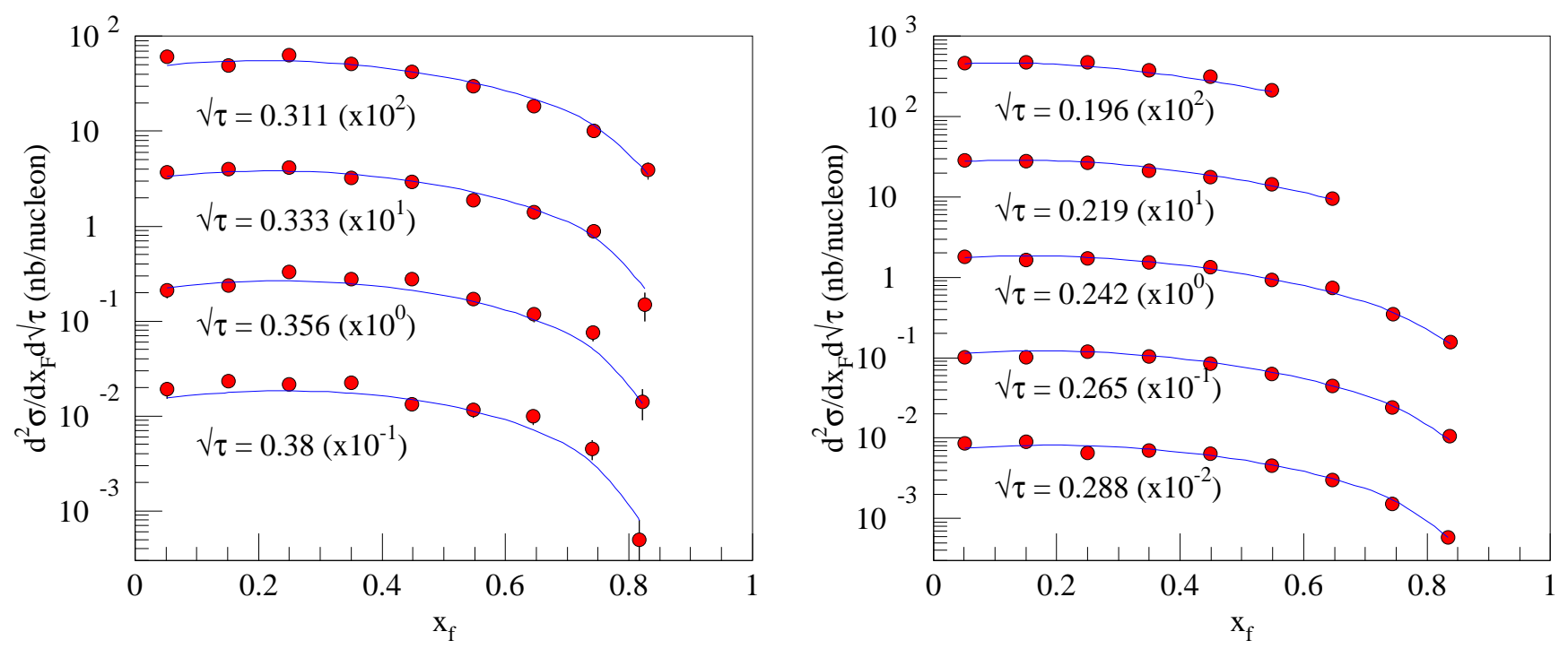

FIG. 2: (Color online) Measured values of $d \sigma / d x_{F} d \sqrt{\tau}$ (points) reported by Conway et al. [1] along with the NLO fit to the data (curves).

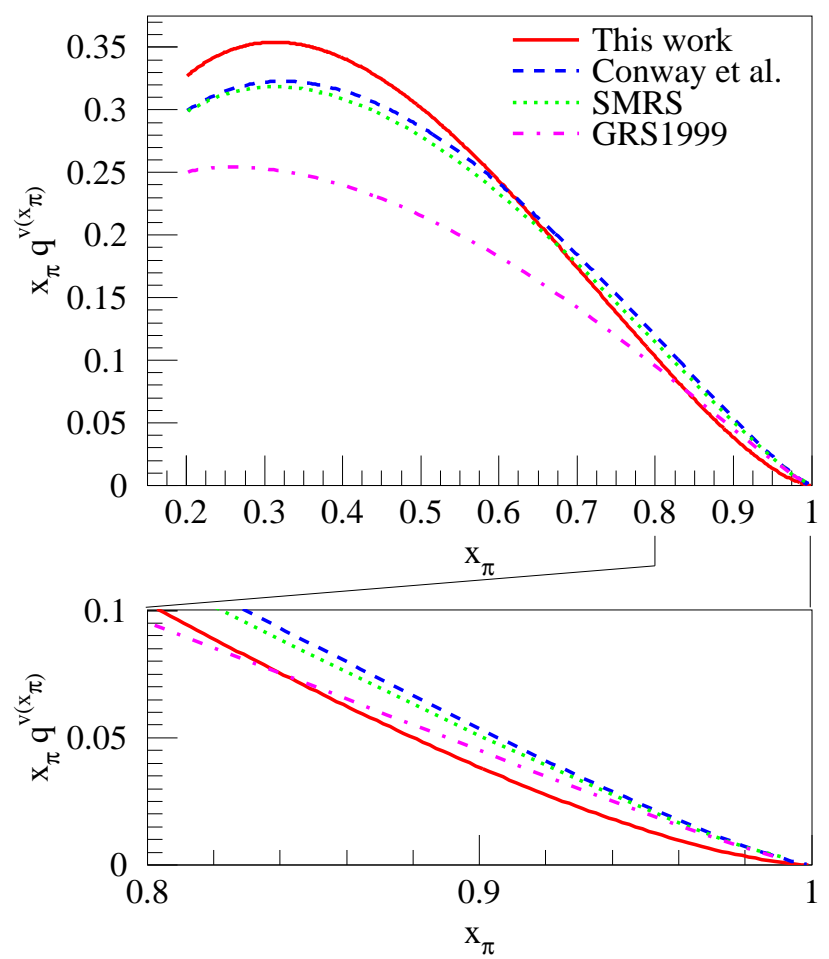

FIG. 3: (Color online) The valence pion PDF, $x q_{\pi}^{v}(x)$, for the preferred fit (solid) as well as those from Conway, et al. [1] (dashed), SMRS 31] (dotted) and GRS 27] (dot-dashed) at a mass scale of $\left\langle M_{\gamma}\right\rangle^{2}=(5.2 \mathrm{GeV})^{2}$ are shown. The present work shows evidence for greater curvature at high- $x$. The upper plot shows the entire $x$ range of the data, while the lower plot emphasized the high- $x$ region. For comparison, the small higher twist $(\gamma)$ term has been removed.
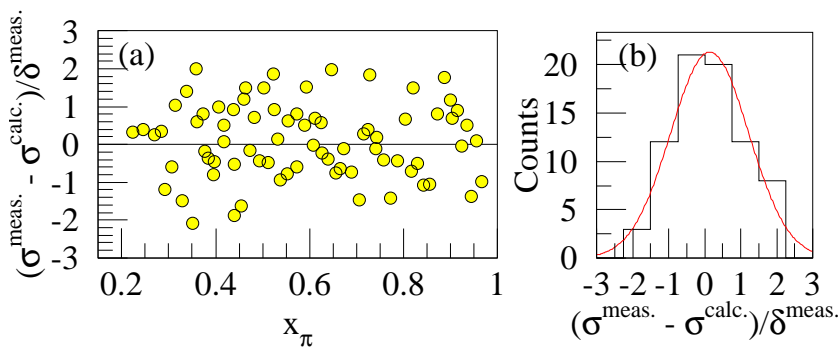

FIG. 4: (Color online) (a) The residuals, $R=$ $\left(\sigma^{\text {meas. }}-\sigma^{\text {calc. }}\right) / \delta^{\text {meas. }}$, for the preferred fit as a function of $x$. No bias is apparent as a function of $x$. (b) The residual distribution fit to a Gaussian with $\langle R\rangle=0.14 \pm 0.15$ and $\sigma=1.09 \pm 0.14$.

TABLE II: The moments of the pion valence PDF, evaluated at a mass scale of $\left\langle M_{\gamma}\right\rangle^{2}=(5.2 \mathrm{GeV})^{2}$, are tabulated below. The $0^{\text {th }}$ moment was a constraint of the fit-see Eq. 8

\begin{tabular}{ll}
\hline \hline $\mathrm{n}$ & $\int_{0}^{1} x^{n} q_{\pi}^{\mathrm{v}}(x) d x$ \\
\hline 1 & $0.217 \pm 0.011$ \\
2 & $0.087 \pm 0.005$ \\
3 & $0.045 \pm 0.003$ \\
\hline \hline
\end{tabular}

flavor symmetric limit-see Eq. 7 to no strange sea. Since most of the data was in the pion's valence region, it is not surprising that the strange sea of the pion had little effect on the valence parameterization.

Several earlier works have also considered the data fit by the present work, as shown in Fig. 3. The fit by Conway et al. was in LO and showed less curvature at high- $x$, 
but Conway even speculated that a NLO fit might yield different results 2]. The fit by SMRS 31] looked at this data as well as other pionic data. While this fit was in NLO, it was based on what is now an old description of the proton's PDFs with a linear, ad hoc nuclear correction. In addition, some of the high- $\tau$ data was excluded from the fit. GRS [27] studied the pionic PDF in NLO with the less restrictive parameterization, but this work also invoked "constituent quark independence" which directly relates the pion and proton distributions [27], introducing a different possible bias.

We have presented a NLO analysis of Drell-Yan data from Fermilab E-615. When analyzed in NLO, the pions valence $\mathrm{PDF}$ clearly have more curvature as $x \rightarrow 1$ than previous leading order fits. This is in worse agreement than previous analysis with the linear dependence expected by NJL models [15, 16, 17, 18], duality argument [21] and the Drell-Yan-West relation [19, 20]. It is not as much as is expected from Dyson-Schwinger Equation based models of the pion 5, 9, 10, 11, 12 or pQCD [7, 8], however. Finally, while E615's resolution in $x$ is not given, the mass resolution for the $J / \psi$ is $\sigma_{M}=0.16 \mathrm{GeV}$ [2]. This could translate into substantial uncertainty in $x$ as $x \rightarrow 1$, so additional data from a more precise experiment with better resolution would certainly be welcome.

We thank C.D. Roberts and W. Melnitchouk for many useful discussions and W. Tung of CTEQ for providing the code necessary for the NLO Drell-Yan cross section calculation. This work was supported by the U.S. Department of Energy, Office of Nuclear Physics, under Contract No. W-31-109-ENG-38.
[1] J. S. Conway et al., Phys. Rev. D39, 92 (1989).

[2] J. S. Conway, Ph.D. thesis, University of Chicago (1987), Fermilab-thesis-1987-16.

[3] J. Badier et al. (NA3), Z. Phys. C18, 281 (1983).

[4] B. Betev et al. (NA10), Z. Phys. C28, 9 (1985).

[5] M. B. Hecht, C. D. Roberts, and S. M. Schmidt, Phys. Rev. C63, 025213 (2001), nucl-th/0008049.

[6] G. R. Farrar and D. R. Jackson, Phys. Rev. Lett. 35, 1416 (1975).

[7] X.-d. Ji, J.-P. Ma, and F. Yuan, Phys. Lett. B610, 247 (2005), hep-ph/0411382.

[8] S. J. Brodsky, M. Burkardt, and I. Schmidt, Nucl. Phys. B441, 197 (1995), hep-ph/9401328.

[9] P. Maris and C. D. Roberts, Int. J. Mod. Phys. E12, 297 (2003), nucl-th/0301049.

[10] J. C. R. Bloch, C. D. Roberts, S. M. Schmidt, A. Bender, and M. R. Frank, Phys. Rev. C60, 062201 (1999), nuclth/9907120.

[11] J. C. R. Bloch, C. D. Roberts, and S. M. Schmidt, Phys. Rev. C61, 065207 (2000), nucl-th/9911068.

[12] M. B. Hecht, C. D. Roberts, and S. M. Schmidt, in Proceedings of the workshop on light-cone $Q C D$ and Nonperturbative hadron physics (1999), nucl-th/0005067.

[13] T. Frederico and G. A. Miller, Phys. Rev. D50, 210 (1994).

[14] A. Szczepaniak, C.-R. Ji, and S. R. Cotanch, Phys. Rev. D49, 3466 (1994), hep-ph/9309284.

[15] T. Shigetani, K. Suzuki, and H. Toki, Phys. Lett. B308, 383 (1993), hep-ph/9402286.

[16] R. M. Davidson and E. Ruiz Arriola, Phys. Lett. B348, 163 (1995).

[17] H. Weigel, E. Ruiz Arriola, and L. P. Gamberg, Nucl. Phys. B560, 383 (1999), hep-ph/9905329.

[18] W. Bentz, T. Hama, T. Matsuki, and K. Yazaki, Nucl. Phys. A651, 143 (1999), hep-ph/9901377.
[19] S. D. Drell and T.-M. Yan, Phys. Rev. Lett. 24, 181 (1970).

[20] G. B. West, Phys. Rev. Lett. 24, 1206 (1970).

[21] W. Melnitchouk, Eur. Phys. J. A17, 223 (2003), hepph/0208258.

[22] A. E. Dorokhov and L. Tomio, Phys. Rev. D62, 014016 (2000).

[23] C. Best et al., Phys. Rev. D56, 2743 (1997), heplat/9703014.

[24] W. Detmold, W. Melnitchouk, and A. W. Thomas, Phys. Rev. D68, 034025 (2003), hep-lat/0303015.

[25] H. L. Lai et al. (CTEQ), Eur. Phys. J. C12, 375 (2000), hep-ph/9903282.

[26] A. D. Martin, R. G. Roberts, W. J. Stirling, and R. S. Thorne, Eur. Phys. J. C4, 463 (1998), hep-ph/9803445.

[27] M. Gluck, E. Reya, and A. Vogt, Eur. Phys. J. C5, 461 (1998), hep-ph/9806404.

[28] C. Adloff et al. (H1), Eur. Phys. J. C6, 587 (1999), hepex/9811013.

[29] S. Chekanov et al. (ZEUS), Nucl. Phys. B637, 3 (2002), hep-ex/0205076.

[30] J. F. Owens, Phys. Rev. D30, 943 (1984).

[31] P. J. Sutton, A. D. Martin, R. G. Roberts, and W. J. Stirling, Phys. Rev. D45, 2349 (1992).

[32] M. Gluck, E. Reya, and I. Schienbein, Eur. Phys. J. C10, 313 (1999), hep-ph/9903288.

[33] K. J. Eskola, V. J. Kolhinen, and C. A. Salgado, Eur. Phys. J. C9, 61 (1999), hep-ph/9807297.

[34] E. L. Berger and S. J. Brodsky, Phys. Rev. Lett. 42, 940 (1979).

[35] M. Bonesini et al. (WA70), Z. Phys. C37, 535 (1988).

[36] J. C. Webb et al. (NuSea) (2003), hep-ex/0302019.

[37] S. Falciano et al. (NA10), Z. Phys. C31, 513 (1986). 